\title{
Optimal Delivery in Display Advertising
}

\author{
[Extended Abstract]
}

\author{
Mohamed Mostagir \\ Social and Information Sciences Laboratory \\ California Institute of Technology \\ mosta@caltech.edu
}

\begin{abstract}
In display advertising, a publisher targets a specific audience by displaying ads on content web pages. Because the publisher has little control over the supply of display opportunities, the actual supply of ads that it can sell is stochastic. We consider the problem of optimal ad delivery, where an advertiser requests a certain number of impressions to be displayed by the publisher over a certain time horizon. Time is divided into periods, and in the beginning of each period the publisher chooses a fraction of the still unrealized supply to allocate towards fulfilling the advertiser's demand. The goal is to be able to fulfill the demand at the end of the horizon with minimal costs incurred from penalties associated with shortage or over-delivery of ads. We describe optimal policies that are both simple in structure and easy to implement for several variations of this problem.
\end{abstract}

\section{INTRODUCTION}

Display advertising has become one of the most profitable areas of online services, responsible for approximately $\$ 24$ billion in business [5]. Unlike sponsored search, where textual ads are displayed along the results of a keyword search, display advertising targets specific audiences by showing graphical banner ads on regular content pages. Targeting can be specific by focusing on certain demographics, so that for example, an ad is only shown to people from a certain age group living in a particular geographic location. Typically, display advertising is handled through direct contracts between the publisher and the advertiser. These contracts are characterized by the publisher committing to the delivery of a pre-specified number of ads to the target audience during a certain time period (also known as guaranteed delivery). Because the supply of display opportunities is uncertain, it is possible that the publisher is unable to fully meet the advertiser's demand, in which case the advertiser is compensated via a penalty (per undelivered impression, for example). Additionally, over-delivering, or providing an advertiser with more impressions than their requested demand can be costly for a variety of reasons. For example, there maybe an op- portunity cost associated with giving the ad away instead of selling it to another advertiser. It is also possible that the advertiser's infrastructure can only handle so many visits from people who see the ad and click on it before that infrastructure breaks down, and so a cap is placed on the number of ads that the advertiser wants displayed during a period of time. The tension between the under and over delivery costs in addition to the stochasticity of the supply is what makes the publisher's problem difficult.

Typically, ads are assigned to advertisers through the use of auctions. Because of the intricacies and complexities of these auctions and the overhead required by the advertisers to handle them, many advertisers simply opt to let the publisher manage their campaigns and do their bidding on their behalf. As in [4], the advertiser indicates a maximum price that it is willing to pay per impression in its contract with the publisher, and the publisher uses this constraint when bidding on impressions for the advertiser. With the volume of traffic generated over the internet, these auctions take place at an incredibly fast rate. It would thus be inefficient, if not completely impossible, to adjust the advertiser's bid after every single auction. Therefore, the advertiser's bid, placed by the publisher, remains effective for a certain period of time until it is re-adjusted for the next time period. By having a constant bid placed over all the auctions taking place in a time period, one can expect to win a fraction of these auctions. We will make use of this correspondence between bids and fractions in our formulation by thinking of our decision variables as fractions of the (uncertain) supply instead of bid values for each time period. This has been the standard approach in recent work on the problem (e.g. [2] and [5]). Like these papers, we think of the supply of ads as a 'channel' with an uncertain capacity. However, unlike the area of literature that focuses on selecting the optimal set of guaranteed contracts (for example, [1], [3], and [4]) to maximize revenue in such a setting, we take the contract as input and focus on how to optimally fulfill the demand under supply uncertainty. We assume that the only control that a publisher exerts over the supply is to decide on a fraction of the channel to allocate towards fulfilling an advertiser's demand before the actual supply is realized for that period. Instead of formulating the problem as that of profit maximization -by fulfilling as much as possible of the demand for the negotiated price per impression- we think of it as a cost minimization problem, where we try to minimize the number of ads not served (equivalent to lost revenue in the maximization model) in addition to the overdelivery penalty 
discussed earlier. The basic question we deal with in this paper is the following: Given an advertiser's demand, a finite planning horizon, and a time-variable supply distribution, how do we choose the fraction of the channel to assign to an advertiser in each period so that our cost at the end of the horizon is minimized under the various penalties? This is similar to some of the questions asked in [2]. In their work, they aimed to give a very general, all-encompassing framework to the problem at the expense of giving solutions that provide no performance guarantees. In contrast to their work, we focus on the specific problem described above and we are able to completely characterize the optimal policy under reasonable assumptions. We also show that while we cannot obtain such a solution for the general case, we can get arbitrarily close to the optimal solution.

Our understanding of online advertising has evolved from looking at the problem as a sequence of seemingly unrelated single-round auctions to become more of a carefully planned campaign that admits more expressive requests from the advertiser's side. For example, as noted earlier, advertisers can be very specific in defining their target groups. In addition, there can be other side constraints or terms added to the publisher's contract. As an example, a contract can specify that, in addition to requiring a certain number of impressions to be delivered over a period of thirty days, the delivery should also be spread as evenly as possible, so that if the demand is, say, 300,000 impressions, then the advertiser would ideally prefer to display 10,000 impressions every day for the duration of the contract. This way the advertiser gets a more steady exposure instead of a possible burst in delivery followed by no advertising that the earlier setting allows (for example, by delivering all ads on the first day and then doing nothing for the rest of the planning horizon). One can easily imagine many ways in which the advertiser can amend their contract to include constraints like the above example. We will give a sufficient condition under which the methods in this paper extend to more expressive contracts.

There is a strong connection between our problem and problems from the theory of stochastic inventory control. The literature in this area is vast, with a standard model of stochastic demand [10] but scattered and problem-specific models for random yield and stochastic supply [9]. Until recently, the focus of this literature has been on identifying the structure of the optimal policies for these problems without much regard to the feasibility of actually computing such policies. Most of these policies were based on dynamic programming formulations and solving the dynamic program was costly and in many cases impossible. Later work was successful in finding approximate policies that either do not rely on dynamic programming [7] or that exploits the structure of the dynamic program to provide near-optimal solutions without the computational burden [6].

The rest of the paper is organized as follows. Section 2 gives a formal definition of the problem, while Section 3 derives the optimal policy to the single advertiser case. Section 4 shows how to extend the solution to the general case with multiple advertisers as well as extensions to more expressive contracts. Section 5 concludes the paper and suggests possible extensions to our work.

\section{MODEL AND NOTATION}

We will highlight some of the methods used throughout the paper by focusing on the single advertiser case for most of this abstract, and so we present the model for this case first. The extension to multiple advertisers is straightforward and will be given in Section 4. We first consider the demand side of the problem. An advertiser requests a number of ads that it would like displayed over a certain time horizon. Time is discrete and is divided into periods, with the planning horizon consisting of $T$ periods. The advertiser wishes to have a total of $D$ impressions delivered over the entire horizon. Later on we will discuss the case when the advertiser can also specify additional requirements, like even spacing of impressions over time, etc.

The supply is stochastic and time-variable. In each time pe$\operatorname{riod} t, t=1, \ldots, T$, the publisher gets a random number $X_{t}$ of display opportunities that are related to the advertiser's target group. Here, $X_{t}$ is a random variable that is distributed according to a known distribution $F_{t}(x)$, with density $f_{t}(x)$. We assume that the supply distributions across periods are independent, but not necessarily identically distributed. In each period $t$ and before $X_{t}$ is realized, the publisher decides on a fraction $\alpha_{t}, 0 \leq \alpha_{t} \leq 1$, to be taken out from the random supply $X_{t}$ in order to fulfill part of the demand $D$. As discussed earlier, this fraction is equivalent to selecting a bid that ultimately awards the advertiser a fraction of the supply at the end of the period. At the end of the planning horizon, the publisher incurs a penalty per undelivered impression, denoted by $p_{1}$. There is also a penalty per overdelivered impression, which can be thought of as the cost of giving away an impression for free instead of selling it. We will denote this penalty by $p_{2}$. At time $T=0$, the expected cost over the planning horizon can be expressed as

$$
E\left[p_{1}\left(D-\sum_{t=1}^{T} \alpha_{t} X_{t}\right)^{+}+p_{2}\left(\sum_{t=1}^{T} \alpha_{t} X_{t}-D\right)^{+}\right]
$$

where $(y-a)^{+}=\max (y-a, 0)$. The publisher's problem is to select the fractions $\alpha_{1}, \ldots, \alpha_{T}$ such that this expected cost is minimized. Put differently, the publisher wants to find a policy whereby given the number of remaining impressions at the beginning of period $t$, it sets the fraction $\alpha_{t}$ such that the optimal expected cost is achieved, assuming that optimal decisions will be made in periods $t+1, \ldots, T$. Note that, perhaps contrary to one's initial intuition, a greedy policy that assigns high fractions to the advertiser in earlier periods is not necessarily optimal since the supply distributions are time variant. In fact, we can show that the following result is true of any myopic policy (which includes the class of all greedy policies).

Lemma 2.1. Any myopic policy for the single advertiser ad delivery problem can perform arbitrarily badly compared to the optimal solution.

A myopic policy by definition does not take the future into account and tries to provide a solution as if the current period is the last or only period in the problem. The following simple example shows that the preceding lemma is true. 
EXAMPLE 2.2. An advertiser has a demand of 40 ads, to be delivered over two periods. The cost of overdelivery is 1 and the shortage cost is 2 . In the first period, the supply of ads is a Bernoulli random variable, taking a value of either 50 or 100 with equal probability. The supply in the second period is again a Bernoulli random variable, taking the value 50 with probability $\epsilon$ and 100 with probability $1-\epsilon$. Denote by $\alpha_{1}$ and $\alpha_{2}$ the fraction of supply assigned to the advertiser in periods 1 and 2, respectively, and let the cost of the myopic policy be Cost $_{\text {myopic }}$ and the cost of the optimal policy

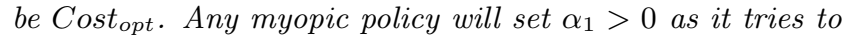
fulfill some of the demand in period 1, and therefore incurs positive expected cost (in fact, for this example a myopic policy that tries to optimally balance overdelivery and shortage costs in the first period sets $\alpha_{1}=0.645$ and incurs an expected cost of 13 in the first period alone). As $\epsilon$ goes to zero however, the optimal solution sets $\alpha_{1}^{*}=0$ and $\alpha_{2}^{*}=0.4$, and the optimal cost approaches zero, making $\frac{\text { Cost }_{\text {myopic }}}{\text { Cost }_{\text {opt }}} \rightarrow \infty$

Obviously, as soon as overdelivery occurs in one period and the associated costs are incurred, there is no reason to assign any future supply to the advertiser. One can think of fulfilling the demand over multiple periods as a chance to avoid overdelivery in any one particular period by spreading the delivery over the entire horizon.

Unsurprisingly, the sequential nature of the problem lends itself to a dynamic programming framework. Let the state variable at time $t$ be $d_{t}$, the number of remaining impressions to be displayed over the rest of the planning horizon. The sequence of events in period $t$ is as follows. $d_{t}$ is observed and the fraction $\alpha_{t}$, is set to some value. The supply $X_{t}$ is then realized and the yield $\alpha_{t} X_{t}$ goes towards fulfilling part or all of the advertiser's demand. The state variable for the next period, $d_{t+1}$, is set equal to $\left(d_{t}-\alpha_{t} X_{t}\right)^{+}$. We will denote by $g_{t}\left(d_{t}\right)$ the optimal expected cost-to-go function; that is, $g_{t}\left(d_{t}\right)$ is the optimal expected cost at time $t$ when there are $d_{t}$ remaining impressions, and assuming that optimal decisions will be made in periods $t$ through $T$.

\section{SINGLE ADVERTISER}

We will start the analysis by focusing on the case of a single advertiser. It is worth nothing that, in addition to the benefits of illustrating the structure of the solution in a simplified context, this case is also of relevant practical interest. In the multiple advertisers case, the advertisers' problems are linked through the constraint that the sum of the fractions of supply assigned to them is at most one. Since in some scenarios it is not uncommon for the publisher to have more supply than the aggregate demand, this constraint becomes non-binding, and the problem can be decoupled into separate single advertiser problems. Taking this view further, we formalize the preceding point in the assumption that follows. Let the optimal fraction in period $t, t=1, \ldots, T$ be denoted by $\alpha_{t}^{*}$ and consider

Assumption 3.1. In the optimal solution to the single advertiser delivery problem, $\alpha_{t}^{*}<1$ for all $t$.

As mentioned, one can easily think of scenarios where this assumption would be valid. Indeed, there will be specific target groups and/or various criteria for which it is probably never the case that the publisher assigns all the display opportunities it gets to a single advertiser, since the advertiser's demand is considerably smaller than the available supply, and hence the optimal fraction of ads assigned to that advertiser will always be strictly less than one (as a trivial example, think of an advertiser that wants to display ads to males in the age bracket of 20 to 40 - a very large target audience). On the other hand, one can construct examples where the optimal solution gives the advertiser every single display opportunity that the publisher gets. This may happen if the advertiser is interested in a very unique set of target demographics, such that the supply of the display opportunities for the specified criteria is scarce and barely enough to fulfill the demand. Another possibility is that the cost per undelivered impression is extremely high compared to the per-impression overdelivery cost, resulting in a very conservative policy that aims to avoid shortage by setting $\alpha$ to its maximum possible value. For the purposes of this section though, and assuming that the above assumption holds, we can derive a simple closed-form for the optimal policy that is summarized in the following theorem. Although we will not prove it here, it is worth mentioning that the optimal policy derived herein is also the unique optimal policy for the problem. This contrasts with many inventory management problems, where sometimes it can even be computationally difficult to count the number of optimal solutions [6].

THEOREM 3.2. Let $d_{t}$ be the number of remaining impressions at the beginning of period $t$. There exists nonnegative numbers $k_{1}, k_{2}, \ldots, k_{T}$, such that in the ad delivery problem, the optimal policy in period $t$ is to set $\alpha_{t}^{*}=d_{t} / k_{t}$. Furthermore, computing the values $k_{t}$ for $t=1, \ldots T$ can be done efficiently in an offline (i.e. before the first period begins) manner.

Proof. We start by solving a single period problem and then extend the solution to its multi-period counterpart. Consider a single period problem with demand $D$ and random supply $X$. A fraction $\alpha^{*}$ is chosen before $X$ is realized such that $\alpha^{*}$ is the solution to the following problem

$$
\min _{\alpha} E\left[p_{1}(D-\alpha X)^{+}+p_{2}(\alpha X-D)^{+}\right]
$$

This expectation can also be written as

$$
p_{1} \int_{0}^{D / \alpha}(D-\alpha x) d F(x)+p_{2} \int_{D / \alpha}^{\infty}(\alpha x-D) d F(x)
$$

which can be verified to be a convex function of $\alpha$. The first derivative of (2) with respect to $\alpha$ is given by

$$
-p_{1} \int_{0}^{D / \alpha} x f(x) d x+p_{2} \int_{D / \alpha}^{\infty} x f(x) d x
$$

Because $x$ is a nonnegative random variable, the integral $\int_{a}^{b} x f(x) d x$ is equal to the integral $\int_{a}^{b}(1-F(x)) d x$. The second derivative, again with respect to $\alpha$ is then equal to

$$
\frac{p_{1} D}{\alpha^{2}} \int_{0}^{D / \alpha} x f(x) d x+\frac{p_{2} D}{\alpha^{2}} \int_{D / \alpha}^{\infty} x f(x) d x
$$

This expression is greater than zero for any nontrivial specification of the problem (i.e. a specification with $p_{1}>0, p_{2}>$ 
$0, D>0$, and a distribution $F(x)$ that does not put all the weight on zero). Hence the function is convex in $\alpha$ and the first order condition for minimization obtained from setting (3) equal to zero tells us that $\alpha^{*}$, the fraction for which the expectation in (1) is minimized, satisfies

$$
\alpha^{*}=\sup _{\alpha}\left\{\frac{\int_{0}^{D / \alpha} 1-F(x) d x}{\int_{D / \alpha}^{\infty} 1-F(x) d x}\right\} \geq \frac{p_{2}}{p_{1}}
$$

where the inequality, instead of equality, accounts for discrete distributions. Recalling that the integral $\int_{a}^{b}(1-F(x)) d x$ for a nonnegative random variable $X$ gives the expectation of $X$ over the interval $(a, b)$, the optimality condition can be interpreted as finding the fraction $\alpha^{*}$ that divides the support of $X$ into two intervals, $\left[0, D / \alpha^{*}\right]$ and $\left(D / \alpha^{*}, \infty\right)$, such that the ratio of the contribution of these two intervals to the expectation of $X$ is equal to the ratio $p_{2} / p_{1}$. In the case of a discrete distribution, $D / \alpha^{*}$ would be the first point in the support of $X$ that makes this ratio equal to or bigger than $p_{2} / p_{1}$. If no such point exists, then $\alpha^{*}$ is set to its maximum value of one, a possibility that we will ignore when we move to the multi-period version under Assumption 3.1.

It is not difficult to see that, for values $p_{1}$ and $p_{2}$ and a certain distribution $F(x)$, there is only one point in the domain of $X$, call it $k$, that would satisfy this ratio condition, i.e. there is a unique value $k$ that solves

$$
k=\inf _{t}\left\{\frac{\int_{0}^{t} x f(x) d x}{\int_{t}^{\infty} x f(x) d x}\right\} \geq \frac{p_{2}}{p_{1}}
$$

Furthermore, computing this point $k$ requires only knowledge of $p_{1}, p_{2}$, and $F(x)$ - it is independent of $D$ and $\alpha$. This implies that we can pre-compute $k$ before $D$ is known and before the problem commences (i.e. we can compute $k$ offline before the period begins). We then use this computed value for $k$ along with the input $D$ to compute $\alpha^{*}=D / k$. Thus the optimal solution to the one period problem can be written as

$$
\alpha^{*}= \begin{cases}D / k, & 0 \leq D / k<1 \\ 1, & D / k \geq 1\end{cases}
$$

Using Assumption 3.1, we write the optimal cost-to-go function, substituting the value of $\alpha^{*}$ from (5) into (2)

$$
g(D)=p_{1} \int_{0}^{k} d\left(1-\frac{x}{k}\right) d F(x)+p_{2} \int_{k}^{\infty} d\left(\frac{x}{k}-1\right) d F(x)
$$

Note that in this expression, the only variable is $d$, by rewriting as

$g(D)=d\left(p_{1} \int_{0}^{k}\left(1-\frac{x}{k}\right) d F(x)+p_{2} \int_{k}^{\infty}\left(\frac{x}{k}-1\right) d F(x)\right)$

we can see that $g(D)$ is a linear function of the form $g(d)=$ $u D$, where $u$ is a nonnegative constant that is equal to $p_{1} \int_{0}^{k}\left(1-\frac{x}{k}\right) d F(x)+p_{2} \int_{k}^{\infty}\left(\frac{x}{k}-1\right) d F(x)$.

Having solved the single period problem, we extend the solution to its multi-period counterpart. Denote the remaining impressions at the beginning of period $T$ by $d_{T}$. Since the problem in period $T$ is identical to the single period problem we just solved, we can find the values $k_{T}$ and $u_{T}$ and write $g_{T}\left(d_{T}\right)=u_{T} d_{T}$. Then, moving backwards in time to period
$T-1$ and writing the optimal cost-to-go function for that period, we have

$$
\begin{array}{r}
g_{T-1}\left(d_{T-1}\right)=\min _{\alpha_{T-1}} E\left[p_{2}\left(\alpha_{T-1} X_{T-1}-d_{T-1}\right)^{+}\right. \\
\left.+g_{T}\left(d_{T-1}-\alpha_{T-1} X_{T-1}\right)^{+}\right]
\end{array}
$$

substituting for $g_{T}\left(d_{T}\right)$ by $u_{T} d_{T}$, this expression becomes

$$
\begin{array}{r}
g_{T-1}\left(d_{T-1}\right)=\min _{\alpha_{T-1}} E\left[p_{2}\left(\alpha_{T-1} X_{T-1}-d_{T-1}\right)^{+}\right. \\
\left.+u_{T}\left(d_{T-1}-\alpha_{T-1} X_{T-1}\right)^{+}\right]
\end{array}
$$

which is of the same form as (1), with $p_{1}$ replaced by $u_{T}$. We can then solve for the optimal $\alpha_{T-1}^{*}$ in the exact same way as before, by finding $k_{T-1}$. The optimal policy in period $T-1$ is then similar to that of a single period problem: if the number of remaining impressions at the beginning of period $T-1$ is $d_{T-1}$, then the optimal solution is to set $\alpha_{T-1}^{*}$ to $d_{T-1} / k_{T-1}$ and the optimal cost-to-go in that period can be written as $g_{T-1}\left(d_{T-1}\right)=u_{T-1} d_{T-1}$. Inductively, we deduce that there are values $k_{T-2}, \ldots, k_{1}$ which can all be computed in the same way as in the single period problem, with the optimal policy in any period given as in the statement of the theorem. Thus the problem reduces to solving a sequence of $T$ single period problems. Again, since the values $k_{t}$ and $u_{t}$ depend only on $p_{1} p_{2}$, and $F\left(x_{t}\right)$, they can be computed offline.

It remains to show that $k_{t}$ and $u_{t}$ can be computed efficiently. Indeed, finding $k_{t}$ amounts to solving an equation in a single variable in the continuous case and is only slightly more difficult than calculating the expectation of a random variable in the discrete case. For the latter, assume that the maximum number of values the random variable $X_{t}$ can take is $m$, and that the probability of $X_{t}=x$ is given by $p(x)$, then finding $k_{t}$ involves nothing more than performing binary search on those $m$ values, where at each step of the search the current value $m_{i}$ is taken as a candidate for $k_{t}$ and the summation $\sum_{i=0}^{m_{i}} x_{i} p\left(x_{i}\right)$ is evaluated and divided by $E(x)-\sum_{i=0}^{m_{i}} x_{i} p\left(x_{i}\right)$ and then compared to $\frac{p_{2}}{p_{1}}$. A straightforward, naive implementation of this method will take time $O(m \log m)$, which is already fast enough for all practical purposes. Computing $u_{t}$ takes $O(m)$ time and is dominated by the time it takes to find $k_{t}$. Repeating the entire procedure for each period, we end up with an overall running time of $O(T m \log m)$.

This result makes intuitive sense, and reinforces the discussion we had after Assumption 3.1. For the one period problem, as $k$ increases with increasing $p_{2}$ or decreasing $p_{1}, \alpha^{*}$ decreases in order to try and protect against overdelivery, which becomes a more costly penalty. Similarly, imagine that $p_{1}$ is very high compared to $p_{2}$, then $k$ takes on smaller values, pushing $\alpha^{*}$ towards one in order to protect against the high cost of under-delivery even when $D$ is not very large.

The multi-period solution gives a nice insight into the structure of the problem. The constant $u_{t}$ for period $t$ can be 
written as

$$
u_{t}=u_{t+1} \int_{0}^{k_{t}}\left(1-\frac{x_{t}}{k_{t}}\right) d F\left(x_{t}\right)+p_{2} \int_{k_{t}}^{\infty}\left(\frac{x_{t}}{k_{t}}-1\right) d F\left(x_{t}\right)
$$

where $u_{T+1}=p_{1}$. From this expression, we can see that $u_{t} \geq u_{t+1}$ for all $t$. One can interpret $u_{t}$ as the cost of waiting to fulfill an impression in the next period instead of the current period. Since $u_{t}$ increases as $t$ decreases, there is a tendency to not wait until the end of the horizon to deliver impressions. Of course, this is not necessarily true for all scenarios as it also depends on the supply distributions throughout the horizon. Consider the case where supply is IID across periods. Obviously, $d_{t} \geq d_{t+1}$ for all $t$, and since $k_{t}$ varies inversely with $u_{t}$ (recall that $u_{t}$ is analogous to $p_{1}$ in (4)), $k_{t}$ decreases as $t$ decreases and $u_{t}$ increases, so that $k_{t} \leq k_{t+1}$ for all $t$. This implies that $\alpha_{t}^{*}=\frac{d_{t}}{k_{t}}$ starts at some value in the first period and then keeps decreasing as $t$ increases, so that in the optimal policy there is a tendency to deliver the majority of impressions early on to protect against under-delivery, and use the later periods mainly to fill in shortages from earlier periods. Given that the solution can be computed knowing only the costs $p_{1}$ and $p_{2}$ and the demand distributions, the publisher can use this information about the optimal cost to adjust and negotiate the penalties $p_{1}$ and $p_{2}$ so that the resulting contract has the minimum possible cost given the demands and requirements of the advertiser.

When Assumption 3.1 is violated, the policy in Theorem 3.2 is no longer optimal. The reason for this is that the dependence of the optimal cost-to-go on $d$ is no longer linear but convex, indicating that we need to evaluate $g_{t}$ for all values of $d_{t}$ to apply backwards induction. When this is the case, the problem has a pseudopolynomial time algorithm with a running time $O(T D)$. The direct dependence on $D$ makes the problem intractable. However, we can still develop a fully polynomial time approximation scheme for the problem. This means that for any given $\epsilon$, we can find a solution that is within $\epsilon$ from the optimal solution and that requires time polynomial in the input size and $1 / \epsilon$ to compute. The proof of this result is quite involved, and can be found in [8].

\section{EXTENSIONS}

\subsection{Multiple Advertisers}

We will slightly revise the structure of the costs before we extend the results to multiple advertisers. Throughout the preceding discussion, we have interpreted the penalty $p_{2}$ as the opportunity cost of giving away an impression for free instead of selling it to another advertiser. When we consider the multiple advertisers case under this interpretation, there is no reason to keep the overdelivery costs, since the case where advertiser $i$ is allocated more impressions than their demand only impacts the solution if this overdelivery results in shortage for other advertisers, and hence the penalty $p_{2}$ can be implicitly incorporated into the shortage costs of advertisers other than $i$. We will discuss the case when the advertiser also wishes to not receive extra impressions over their demand in the next subsection. For this section, we assume that there are $m$ advertisers and that advertiser $i$ 's shortage cost is given by $p_{i}$. The decision vector in period $t$ is $\alpha^{\mathbf{t}}=\left(\alpha_{1}^{t}, \ldots, \alpha_{m}^{t}\right)$, where $\alpha_{i}^{t}$ is the fraction assigned to advertiser $i$ in period $t$. The problem then is the same as before: we are interested in choosing $\alpha^{\mathbf{t}}, t=1, \ldots, T$ in order to minimize the shortage costs at the end of the horizon. One difference is that all cost is evaluated at the last period, since there is no longer an overdelivery cost in any one period. Formally,

$$
\begin{array}{rl}
\min _{\alpha^{\mathrm{t}}} & E\left[\sum_{i} p_{i}\left(d_{i}-\sum_{t} \alpha_{i}^{t} x^{t}\right)^{+}\right] \\
\mathrm{s.t.} & \sum_{i} \alpha_{i}^{t} \leq 1 \quad t=1, \ldots, T \\
& 0 \leq \alpha_{i}^{t} \leq 1 \quad t=1, \ldots, T .
\end{array}
$$

We will assume that the constraints $\sum_{i} \alpha_{i}^{t} \leq 1$ are binding. This is without loss of generality, since one can introduce a dummy advertiser that gets assigned any leftover impressions in a period if the constraint has some slack. Furthermore, if in the optimal solution we have $\sum_{i} \alpha_{i}^{*^{t}}<1$ for all $t$, then the problem can simply be decoupled into $m$ separate problems that are then solved as in the previous section. Starting again from the one period problem, we can verify convexity in $\alpha$ as in the single advertiser case. The constraints are linear in $\alpha_{1}, \ldots, \alpha_{m}$ and the Hessian matrix of the objective is positive definite.

Under the binding constraints assumption, advertiser $m$ is assigned a fraction $1-\sum_{i \neq m} \alpha_{i}$, so that setting the fractions for all but the last advertiser automatically determines the fraction that the last advertiser gets. Rewriting the singleperiod objective in the form of (2), we get

$$
\begin{array}{r}
p_{1} \int_{0}^{d_{1} / \alpha_{1}}\left(d_{1}-\alpha_{1} x\right) d F(x)+\ldots \\
+p_{m} \int_{0}^{\frac{d_{m}}{1-\sum_{i \neq m} \alpha_{i}}}\left(d_{m}-\left(1-\sum_{i \neq m} \alpha_{i}\right) x\right) d F(x)
\end{array}
$$

Notice that when minimizing (6), we end up with a system of $m-1$ equations, corresponding to the $m-1$ decision variables $\alpha_{1}, \ldots \alpha_{m-1}$. Since each equation is the partial derivative of (6) with respect to one of the variables, it has exactly two terms: the derivative of the integral that contains that variable as well as the derivative of the last integral, which is expressed in terms of the first $m-1$ variables. Specifically, the derivative of (6) with respect to $\alpha_{i}$ is given by

$$
-p_{i} \int_{0}^{d_{i} / \alpha_{i}} x f(x)+p_{m} \int_{0}^{\frac{d_{i}}{1-\sum_{i \neq m \alpha_{i}}}} x f(x)
$$

Note that in particular, the second term is common to all equations. Writing this out for all the $m-1$ variables and equating each derivative to zero to obtain the conditions for minimization, we find that, for any two advertisers $i$ and $j$, the following holds at the optimal solution

$$
p_{i} \int_{0}^{d_{i} / \alpha_{i}} x f(x)=p_{j} \int_{0}^{d_{j} / \alpha_{j}} x f(x)
$$

Like before, we will let $k_{i}=\frac{d_{i}}{\alpha_{i}}$. The optimal solution to the problem then involves finding $k_{1}, \ldots, k_{m-1}$ such that Condition (6) is satisfied for all $i$ and $j$. In addition, since determining $k_{i}, i=1, \ldots, m-1$ determines $\alpha_{i}, i=1, \ldots, m-1$, it also determines $\alpha_{m}$ through the relation $\alpha_{m}=1-\sum_{i \neq m} \alpha_{i}$. The resulting $\alpha_{m}$ should satisfy Condition (6). Without loss of generality, let the costs $p_{i}$ be arranged such that 
$p_{1} \geq p_{2} \geq \ldots \geq p_{m}$. If we follow the approach from the previous section, we can try to find values of $k_{i}$ such that the following holds for all $i$ and $j$

$$
\frac{\int_{0}^{k_{i}} x f(x)}{\int_{0}^{k_{j}} x f(x)}=\frac{p_{j}}{p_{i}}
$$

A set of values for $k_{i}, i=1, \ldots m$ that solves (7) and leads to a vector $\alpha$ with $\sum_{i} \alpha_{i}=1$ gives a solution to the problem. From (7) and the fact that $X$ is a nonnegative random variable, we can see that advertisers with low index have lower $k$ values. The immediate implication is that these advertisers get more share of the supply if the demands of all advertisers are the same or comparable (since low $k$ values correspond to high values for $\alpha$ when the demands are the same). This agrees with intuition and suggests that the optimal single period policy has a greedy flavor, allocating more shares to those advertisers that have higher penalties. In fact, it is possible that advertisers with high indexes (low $p_{i}$ ) get assigned zero impressions, since the only way the condition is satisfied is if their corresponding values of $k_{i}$ are set to infinity. Of course, since the conditions above also depend on $d_{i}$, it is not always the case that high index advertisers receive less impressions - the important thing is that the optimality conditions are satisfied.

When we consider the multiple period problem, applying the same policy in a myopic fashion turns out to be optimal: at the beginning of each period, we solve the problem as if it is simply a single period problem, and assign to each advertiser the fraction they would get if this was the only period left. While this may seem initially surprising given that we have seen that myopic policies can be arbitrarily bad in Example 2.2, the reason it works in this setting is the removal of the overdelivery cost, which alters the problem and therefore the structure of the optimal policy. A proof for the optimality of the policy above can be found in [8], but the informal reasoning is as follows. Let us think about two advertisers with penalties $p_{1}$ and $p_{2}$ with $p_{1}>p_{2}$ and two periods. Because the myopic policy is greedy by nature, the only way applying it can hurt the publisher is when the optimal solution assigns a bigger share to the second advertiser in the first period than the myopic policy does. But this would only happen when the supply in the second period allows the publisher to reduce the optimal one-period share for the first advertiser in the first period, knowing that it will be able to fulfill the shortage in the second period, but if that is the case then the publisher can just as well use the second period to fulfill the second advertiser's left-over demand instead of taking it out of the (more costly) first advertiser's share in the first period.

\subsection{Additional Delivery Constraints}

Let us return to the single advertiser case. So far, the publisher's problem has been of the form

$$
\min _{0 \leq \alpha_{t} \leq 1} \sum_{t} h\left(d_{t}, F_{t}(x), \alpha_{t}\right)
$$

with $h(d, F(x), \alpha)$ taking the form of the function in (1). We want to consider allowing the advertiser to have more input into the structure of the delivery process, specifically, the advertiser can choose a function $l\left(d_{t}, F_{t}(x), \alpha_{t}\right)$ such that the publisher's objective becomes

$$
\min _{0 \leq \alpha_{t} \leq 1} \sum_{t} h\left(d_{t}, F_{t}(x), \alpha_{t}\right)+l\left(d_{t}, F_{t}(x), \alpha_{t}\right)
$$

Let us illustrate this in the context of the example used at the beginning of the paper, where in addition to the guaranteed delivery requirement, the advertiser would like its ads to be evenly spaced over time. An advertiser with total demand $D$ over a horizon of length $T$ can then choose $l\left(d_{t}, F_{t}(x), \alpha_{t}\right)=q\left|\alpha_{t} x_{t}-\frac{D}{T}\right|$, so that there is a penalty $q$ associated with delivering more or less than $D / T$ impressions in each period (of course, the advertiser can specify any other value than $D / T$, or different values for different periods). For simplicity, let us roll the costs $p_{1}$ and $p_{2}$ into a single cost $p$. The publisher's problem then becomes

$$
\min _{0 \leq \alpha_{t} \leq 1} E\left[p\left|D-\sum_{t=1}^{T} \alpha_{t} X_{t}\right|+q\left|\sum_{t=1}^{T} \alpha_{t} X_{t}-\frac{D}{T}\right|\right]
$$

This problem closely follows the framework outlined above, both for the special case under Assumption 3.1 and the general case (depending on the relationship between $p$ and $q$, it may be necessary to set $\alpha$ equal to 1 in some scenarios). Just to illustrate, under Assumption 3.1 the optimal $\alpha$ in a single period problem satisfies

$$
\alpha^{*}=\sup _{\alpha} \frac{\int_{\frac{D \alpha}{T}}^{\infty} x f(x) d x-\int_{0}^{\frac{D \alpha}{T}} x f(x) d x}{\int_{0}^{\frac{D}{\alpha}} x f(x) d x-\int_{\frac{D}{\alpha}}^{\infty} x f(x) d x}
$$

As one can tell from this expression, the criteria for optimality looks more complex as one adds more requirements. Nevertheless, the structure of the solution (finding intervals that divide the domain of the distribution in a certain way) remains intact. It turns out that a sufficient condition to add more expressiveness while maintaining the general flavor of the solution is the requirement that $l\left(d_{t}, F_{t}(x), \alpha_{t}\right)$ be convex, which makes the publisher's overall objective convex in $\alpha_{t}$ and $d_{t}$. If $l\left(d_{t}, F_{t}(x), \alpha_{t}\right)$ is chosen such that, for example, there is a bonus paid to the publisher once a certain target $z<D$ is fulfilled, then the objective displays a kink and convexity is destroyed. In such scenario, the methods outlined in this paper may fail to be optimal.

\section{CONCLUSIONS}

We have given optimal policies to some variants of the guaranteed ad delivery problem in display advertising. We have seen that when the advertiser's demand is low compared to the overall supply, the problem can be solved to optimality and the optimal policy has a nice and simple characterization. Because the publisher is able to calculate its expected cost as a function of the demand $D$ and costs $p_{1}$ and $p_{2}$, it can use this information in deciding on prices to charge the advertiser for service, as well as negotiate the shortage penalty $p_{1}$. The case for multiple advertisers maintains the same spirit of the solution, namely, dividing the support of the distribution into intervals from which the optimal fractions can be calculated. While figuring out the fractions for the single period multiple advertisers case is not as straightforward as the single advertiser one, the difficulty turns out to be balanced by the fact that a myopic policy is optimal for the multiple advertisers case. If instead of the modification we introduced in the multiple advertiser scenario we 
had each advertiser still maintain under and over delivery penalties then a myopic policy is no longer optimal and the problem becomes quite difficult even to approximate.

There are many variations on the theme of this problem. We have already discussed a sufficient condition under which the methods presented here extend to more expressive contracts, namely, the convexity of the publisher's objective function. It would be interesting to identify the correspondence between bids and fractions: we know what fraction the publisher should set in the optimal solution to the problem, but in reality, and as mentioned in the introduction, the publisher places a bid in an auction for a period of time, not a fraction. The interaction between maximum prices that advertisers are willing to pay per impression and the bids placed by the publisher affects the fractions that the advertiser can select and therefore the structure of the optimal delivery policies. It would therefore be instructive to understand how the two separate processes of selecting optimal contracts and fulfilling these contracts interact so that instead of designing each in isolation one can develop a more integrated approach that accounts for the issues addressed by each.

\section{REFERENCES}

[1] M. Babaioff, J. Hartline, and R. Kleinberg. Selling banner ads: Online algorithms with buyback. In Fourth Workshop on Ad Auctions, 2008.

[2] C. Boutilier, D. Parkes, T. Sandholm, and W. Walsh. Expressive banner ad auctions and model-based online optimization for clearing. In Proceedings of National Conference on Artificial Intelligence (AAAI), 2008.

[3] F. Constantin, J. Feldman, S. Muthukrishnan, and M. Pál. Online ad slotting with cancellations. In 4 th Workshop on Ad Auctions, 2008.

[4] U. Feige, N. Immorlica, V. Mirrokni, and H. Nazerzadeh. A combinatorial allocation mechanism with penalties for banner advertising. 2008.

[5] A. Ghosh, P. McAfee, K. Papineni, and S. Vassilvitskii. Bidding for representative allocations for display advertising. Internet and Network Economics, pages 208-219, 2009.

[6] N. Halman, D. Klabjan, M. Mostagir, J. Orlin, and D. Simchi-Levi. A Fully Polynomial-Time Approximation Scheme for Single-Item Stochastic Inventory Control with Discrete Demand. Mathematics of Operations Research, 34(3):674-685, 2009.

[7] R. Levi, M. Pal, R. Roundy, and D. Shmoys. Approximation algorithms for stochastic inventory control models. Mathematics of Operations Research, 32(2):284, 2007.

[8] M. Mostagir. Optimal Delivery in Display Advertising. 2010. Working paper.

[9] C. Yano and H. Lee. Lot sizing with random yields: a review. Operations Research, pages 311-334, 1995.

[10] P. Zipkin. Foundations of inventory management. 2000. 